\title{
Robust Optimal Design of Fiber-Reinforced Composite Cylinder with Metallic Liner
}

\author{
Chwei-Yuh Chiou \\ Chung Shan Institute of Science \& Technology, P.O. Box 90008-15-3, Lungtan, Taoyuan County 325, Taiwan \\ Correspondence should be addressed to Chwei-Yuh Chiou, chioucy7638@yahoo.com.tw
}

Received 14 May 2012; Accepted 8 July 2012

Academic Editors: D. Aggelis, C. F. Gao, and F. H. Moukalled

Copyright () 2012 Chwei-Yuh Chiou. This is an open access article distributed under the Creative Commons Attribution License, which permits unrestricted use, distribution, and reproduction in any medium, provided the original work is properly cited.

\begin{abstract}
An enhanced robust optimal design method dealing with both property and material uncertainties is established in this paper. The method is applied to the robust optimal design of fiber-reinforced composite cylinder with reinforced composite patches and metallic liner subjected to uniform pressure and local loadings. Instead of the traditional numerical approach, modified constraints are used to analytically solve the antioptimization subproblem by a maximizing procedure in the developed method, consequently the computation time for robust optimal design problem can be significantly reduced. The effectiveness and accuracy of the developed method are verified by a degenerated example. Analysis result shows the optimal weight increases significantly with property and material uncertainties as expected. It is also found that the optimal thickness of metallic liner is affected by the utilized design rules of metallic liner. For plastic design, the thickness of metallic liner should be kept as small as possible for a minimal weight optimal design provided that manufacturing and nonleakage constraints can be met. On the contrary the optimal thickness of metallic liner depends on the relative ratio of allowable strain of metallic liner and composite material if elastic design is used.
\end{abstract}

\section{Introduction}

The anisotropic behavior of fiber-reinforced composite structure can be brought into beneficial usage by tailoring their properties through selection of orientations, thickness, and ply ratios $[1,2]$. This provides the structural designer with a wide range choice of mechanical properties. Properly designed filament winding composite cylinders are widely used for pressure vessels and main sections of hollow-slender shaped aerospace structures with the main advantage of lightweight over their metallic counterparts [3, 4]. Being composed of hoop and helical layers which with the characteristic of unidirectional ply, matrix crack-induced leakage may occur at a stress level much lower than the ultimate strength of fiber in filament wound composite pressure vessel applications. The leakage can be suppressed by the using of inner rubber liner $[5,6]$; however rubber liner has little contribution to the stiffness and strength of the vessel. Some studies utilized metallic liner instead of rubber liner to suppress the leakage of composite cylinder [7-9] since its elongation is much larger than the composite material, and its stiffness and strength are much larger than rubber.

The problem of optimal design of composites is to select the layup arrangement, such as thickness and ply orientation, so as to achieve the highest performance while satisfying specified requirements. Due to the uncertainties of material properties and the variations of ply thickness and orientation in manufacturing, the practical design properties can be different from the prediction of designer. Traditionally, these uncertainties are taken care of via safety factors [1]. However, reliable and efficient safety factors are difficult to obtain since the requested safety factors change case by case. Probabilistic optimal method [10-12] and antioptimization technique [13-15] are two commonly used approaches of optimization for problems with uncertainties. For the former approach, probabilistic analysis techniques such as Mote Carlo simulation, first-order reliability method, and probabilistic finite element method have been applied to evaluate the reliability of composite laminate. Many data are required for an accurate probabilistic model. Large errors 
can be incurred in the calculation of failure probability when such data are inaccurate [16]. A two-level optimization problem is formulated in the traditional anti-optimization technique [13-15]. At the upper level, design variables for the best design (optimization sub-problem) are obtained; antioptimization (anti-optimization sub-problem) for uncertainties is carried out to seek the worst condition for a given design at the lower level. Iterations between upper level and lower level are necessary to obtain the convergent solution. It is apparent that the antioptimum problem is time consuming, and its applications are limited to simple problems with small number of design variables.

Using constraints with sensitivities; that is, uncertainties are taken into account by adding extra sensitivity terms to the traditional constraints, an efficient method was proposed in author's previous work to easily deal with the robust optimization problems of composite laminate with designvariable uncertainties such as ply thickness and ply orientation [17]. Unlike traditional optimal design problems where sensitivities are only used to determine the most appropriate climbing direction in the optimization algorithm, they also serve as the media to evaluate the effects of uncertainty in the proposed method. Basically, the proposed method applies the similar concept of traditional anti-optimization technique, so the only data needed are lower bounds and upper bounds of design variables. Besides the advantage of less data, the proposed method also has the advantage over traditional anti-optimization technique in that the antioptimization sub-problem can be solved analytically rather than numerically via the use of the modified constraints.

In this paper an enhanced method to deal with both property (design-variable) uncertainties and material (nondesign-variable) uncertainties is developed. It is utilized to study the robust optimal designs of fiber-reinforced composite cylinder with metallic liner subjected to uniform pressure and local loadings. Reinforced composite patches [18] are utilized in location where local loading is applied. The effectiveness and accuracy of proposed method are verified through simplified case with analytical solution. The optimal layup of hoop layers, helical layers, and reinforced patches are analyzed. The effects of ply thickness, orientation, and material uncertainties under a variety of metal thickness are investigated, and the effect of two different design approaches, that is, elastic and plastic designs of metallic liner, on the optimal design is thoroughly studied.

\section{Formulation of the Optimization Problem}

For a traditional optimization problem, the basic statements of optimization problem can be written as follows:

$$
\begin{array}{ll}
\text { Minimize } & F(\vec{x}) \text { Objective } \\
\text { Subject to } & g_{j}(\vec{x}, \vec{M}) \leq 0, \quad j=1 \cdots n_{g} \\
& \text { Inequality constraints }
\end{array}
$$

$$
\begin{gathered}
x_{i}^{l} \leq x_{i} \leq x_{i}^{u} \quad i=1, \ldots n \\
\text { Side constraints, } \\
\text { where } \quad \vec{x}=\left\{x_{1}, x_{2} \cdots x_{n}\right\} \text { Design variables. }
\end{gathered}
$$

The objective function $F(\vec{x})$ is the quantity to be minimized. It is a function of the design variables $\vec{x}$. Although it is a minimizing task, we can easily maximize a function by minimizing its negative. Side constraints are placed on the design variables. The inequality constraints are expressed in a less than or equal to zero form by convention. The objective and constraints may be either linear or nonlinear functions.

Being a function of design variables $(\vec{x})$ and material properties $(\vec{M})$, the Taylor series approximation of inequality constraint $g_{j}$ for problems with design variable and material property uncertainties in the neighborhood of $\vec{x}$ and $\vec{M}$ can be described as

$$
\begin{aligned}
g_{j}(\vec{x} & +\overrightarrow{\delta x}, \vec{M}+\delta \vec{M}) \\
= & g_{j}(\vec{x}, \vec{M})+\sum_{i=1}^{n} \delta x_{i} * S_{j i}^{d}+\sum_{i=1}^{m} \delta M_{i} * S_{j i}^{m} \\
& + \text { higher-order terms, }
\end{aligned}
$$

where in the above equation, $\delta x_{i}$ is the uncertainty of design variable $x_{i}, \delta M_{i}$ is the uncertainty of material property $M_{i}, S_{j i}^{d}$ is the sensitivity of $j$ th inequality constraint $g_{j}$ with respect to $i$ th design variable $x_{i}$, and $S_{j i}^{m}$ is the sensitivity of $j$ th inequality constraint $g_{j}$ with respect to $i$ th material property $M_{i}$. Based on our previous work where only property uncertainty was considered [17], and neglecting high order terms for the inequality constraints, the optimization problem with both property and material uncertainties can be formulated as follows:

$$
\begin{array}{ll}
\text { Minimize } & F(\vec{x}) \text { Objective } \\
\text { Subject to } \quad & g_{j}(\vec{x}, \vec{M})+\sum_{i=1}^{n} \delta x_{i} *\left|S_{j i}^{d}\right|+\sum_{i=1}^{m} \delta M_{i} *\left|S_{j i}^{m}\right| \leq 0 \\
& j=1, \ldots n_{g} \text { Inequality constraints } \\
& x_{i}^{l} \leq x_{i} \leq x_{i}^{u}, \quad i=1, \ldots n \text { Side constraints, } \\
& \vec{x}=\left\{x_{1}, x_{2} \cdots x_{n}\right\} \text { Design variables } \\
\text { where } & \delta \vec{x}=\left\{\delta x_{1}, \delta x_{2} \cdots \delta x_{n}\right\} \\
& \text { Uncertainties of design variables } \\
& \delta \vec{M}=\left\{\delta M_{1}, \delta M_{2} \cdots \delta M_{m}\right\} \\
& \text { Uncertainties of material properties. }
\end{array}
$$

The absolute values of design variable and material property sensitivities are used in (4); hence, maximum constraints can be found among all the possible combinations 
of design variables and material properties with maximum uncertainty. Note that each design variable and material property has two extreme values, the maximum and the minimum, under the effect of uncertainty. Hence, there are $2^{n+m}$ extreme combinations for case with $\mathrm{n}$ design variables and $\mathrm{m}$ material properties. The left side of (4) is used to calculate the maximum constraints among the $2^{n+m}$ cases.

Higher-order terms have been neglected in (4); therefore, the error due to the neglected terms increases with the maximum uncertainty. Fortunately, the maximum uncertainties for many engineering problems are small enough for an accurate approximation of (4). It is noted that design variables and material properties may change randomly between lower and upper bounds of uncertainty; hence, there are infinite combinations of those random design variables and material properties, and among them there are $2^{n+m}$ extreme cases. Under the linear approximation, the inequality constraints of (4) are the maximum constraints for any random combinations of design variables and material properties. Similar to the basic concept of antioptimization technique, the uncertainties of design variables are taken care of by a maximizing procedure. Specifically, the uncertainties and sensitivities are combined into the modified constraints. It is more efficient than the antioptimization technique since a time-consuming numerical anti-optimization algorithm must be used in the traditional anti-optimization technique.

\section{Problem Description and Program Implementation}

The geometry of analyzed composite cylinder is shown in Figure 1. Its length and inner diameter are $2.0 \mathrm{~m}$ and $1.0 \mathrm{~m}$, respectively. The occupied circumferential angles and lengths of the three reinforced patches $\left(\varphi_{1}, L_{1}\right),\left(\varphi_{2}, L_{2}\right)$, and $\left(\varphi_{3}, L_{3}\right)$ are $\left(50^{\circ}, 0.5 \mathrm{~m}\right),\left(30^{\circ}, 0.3 \mathrm{~m}\right)$, and $\left(10^{\circ}, 0.125 \mathrm{~m}\right)$. A uniform inner pressure of $4.9 \mathrm{~N} / \mathrm{mm}^{2}$ (load case 1) and local loading of circumferential force of $9800 \mathrm{~N}$ and axial moment of $392 \mathrm{Nm}$ (load case 2) are considered. The local loading is uniformly distributed in the central $100 \mathrm{~mm}$ of patch 3 (circumferential force density $98 \mathrm{~N} / \mathrm{mm}$, axial moment density $3.92 \mathrm{Nm} / \mathrm{mm}$ ) along axial direction. The two loading cases are applied on the composite cylinder separately rather than simultaneously. The extent of patch 1 is chosen so that almost same value of failure index in the perimeter of patch 1 exists when the cylinder is subjected to the two abovementioned loadings separately.

The composite cylinder is composed of SAE4130 inner liner, T300/N5208 helical and hoop layers, and three reinforced patches of T300/Fiberite 934. There are 4 differential layups in the composite cylinder:

layup at location of patch 3: $\left[S_{t s} / \pm \theta_{m 1} / 90_{m 2} /\right.$ $\left.C_{n 1} / C_{n 2} / C_{n 3}\right]$,

layup at location of patch 2: $\left[S_{t s} / \pm \theta_{m 1} / 90_{m 2} /\right.$ $\left.C_{n 1} / C_{n 2}\right]$,

layup at location of patch 1: $\left[S_{t s} / \pm \theta_{m 1} / 90_{m 2} / C_{n 1}\right]$, layup at other locations $\left[S_{t s} / \pm \theta_{m 1} / 90_{m 2}\right]$.

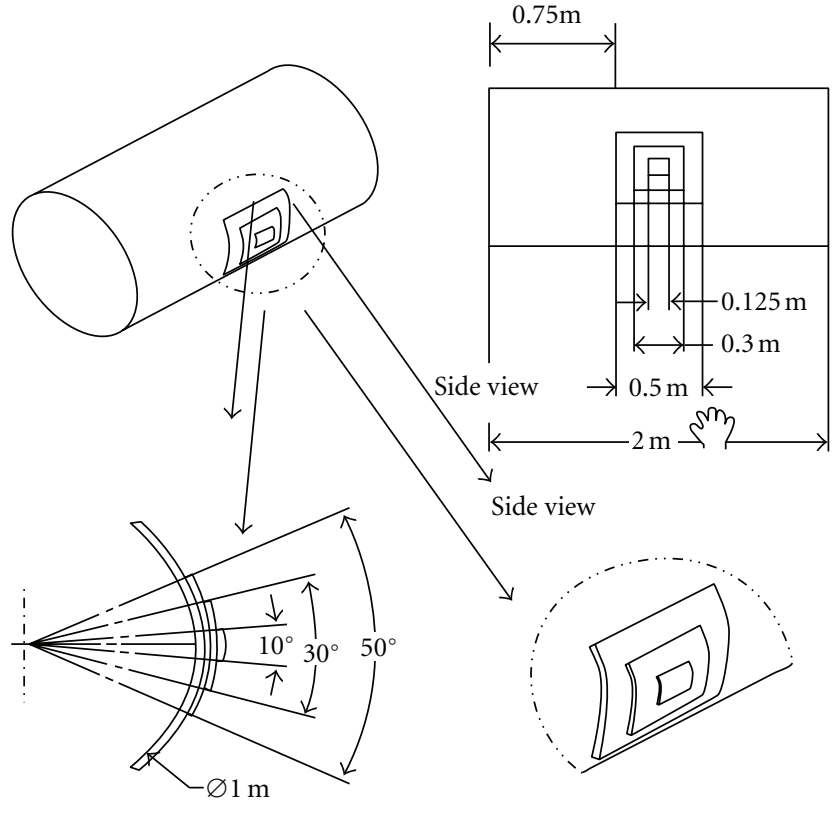

Front view

Detail configuration of reinforced patches (patch $1 \sim 3$ )

FIGURE 1: Geometry of analyzed composite cylinder.

Where $S_{t s}$ denotes SAE4130 of thickness $t s, \pm \theta_{m 1}$ is $m 1$ helical layers of winding angle $\theta$, and $90_{m 2}$ is $m 2$ hoop layers, $C_{n 1}$ is $n 1$ layers of T300/Fiberite 934 cloth in patch $1, C_{n 2}$ is $n 2$ layers of T300/Fiberite 934 cloth in patch 2, and $C_{n 3}$ is $n 3$ layers of T300/Fiberite 934 cloth in patch 3.

The nominal material properties [7] and stiffness uncertainties of T300/N5208 and T300/Fiberite 934 are shown in Table 1 . The stiffness uncertainties, including longitudinal stiffness, transverse stiffness, and in-plane shear modulus uncertainties, of T300/N5208 and T300/Fiberite 934 are assumed to be $10 \%$ of their nominal values. The Young's modulus, the Poisson ratio, yielding stress, and elongation of SAE4130 are $196000 \mathrm{~N} / \mathrm{mm}^{2}, 0.32,980 \mathrm{~N} / \mathrm{mm}^{2}$, and $5 \%$ [19]. The nominal ply thicknesses of T300/N5208 and T300/Fiberite 934 are $0.2 \mathrm{~mm}$; their ply thickness uncertainties are assumed to be $0.02 \mathrm{~mm}$ (10\% of their nominal values), and ply-orientation uncertainty of $5^{\circ}$ is assumed [20].

The object of the problem is to solve the optimal layups of composite cylinder with minimal weight for a variety of thickness of inner liner of SAE4130 subjected to both uniform pressure and local loadings. The design variables are the nominal thicknesses $\left(t_{1} \sim t_{5}\right)$ of helical layer, hoop layer, patch 1, patch 2 , and patch $3\left(t_{1}=0.2 * m 1, t_{2}=0.2 * m 2\right.$, $t_{3}=0.2 * n 1, t_{4}=0.2 * n 2$, and $\left.t_{5}=0.2 * n 3\right)$, and the orientation angle of helical winding $\theta$. Maximum strain failure criterion [7] is used for failure prediction, and the 
TABLe 1: Mechanical properties of T300/N5208 and T300/Fiberite 934.

\begin{tabular}{lccc}
\hline Symbol & Description & T300/N5208 & T300/Fiberite 934 \\
\hline$E_{1}$ & Long. stiffness & $181000.0 \pm 18100.0 \mathrm{~N} / \mathrm{mm}^{2}$ & $74000.0 \pm 7400.0 \mathrm{~N} / \mathrm{mm}^{2}$ \\
$E_{2}$ & Trans. stiffness & $10300.0 \pm 1030.0 \mathrm{~N} / \mathrm{mm}^{2}$ & $74000.0 \pm 7400.0 \mathrm{~N} / \mathrm{mm}^{2}$ \\
$G_{s}$ & In-plane shear modulus & $7170.0 \pm 717.0 \mathrm{~N} / \mathrm{mm}^{2}$ & $4550.0 \pm 455.0 \mathrm{~N} / \mathrm{mm}^{2}$ \\
$v_{12}$ & Long. Poison's ratio & 0.28 & 0.05 \\
$X$ & Long. tensile strength & $1500.0 \mathrm{~N} / \mathrm{mm}^{2}$ & $499.0 \mathrm{~N} / \mathrm{mm}^{2}$ \\
$X^{\prime}$ & Long. compression strength & $1500.0 \mathrm{~N} / \mathrm{mm}^{2}$ & $352.0 \mathrm{~N} / \mathrm{mm}^{2}$ \\
$Y$ & Trans. tensile strength & $40.0 \mathrm{~N} / \mathrm{mm}^{2}$ & $458.0 \mathrm{~N} / \mathrm{mm}^{2}$ \\
$Y^{\prime}$ & Trans. compression strength & $246.0 \mathrm{~N} / \mathrm{mm}^{2}$ & $352.0 \mathrm{~N} / \mathrm{mm}^{2}$ \\
$S$ & In-plane shear strength & $68.0 \mathrm{~N} / \mathrm{mm}^{2}$ & $46.0 \mathrm{~N} / \mathrm{mm}^{2}$ \\
$\gamma$ & Specific weight & 1.6 & 1.5 \\
\hline
\end{tabular}

safety factor must be larger than 1.5 for static and buckling analyses. The optimization statements are as follows:

Minimize $W(\vec{x})$ Objective

Subject to $1.5-\left(\mathrm{S} . \mathrm{F}-\sum_{i=1}^{6} \delta x_{i} *\left|S_{s i}^{d}\right|-\sum_{j=1}^{6} \delta M_{j} *\left|S_{s j}^{m}\right|\right)$ $\leq 0$ Inequality constraints

$1.5-\left(\right.$ Eigen $\left.-\sum_{i=1}^{6} \delta x_{i} *\left|S_{e i}^{d}\right|-\sum_{j=1}^{6} \delta M_{i} *\left|S_{e j}^{m}\right|\right)$

$\leq 0$

$-0 \leq \theta \leq 90$ Side constraints

$0.2 \leq t_{i} \leq 6.0, \quad(i=1 \sim 5)$

where $\vec{x}=\left(t_{1}, t_{2}, \cdots t_{5}, \theta\right)$ Design variables

$\delta \vec{x}=\left(0.1 t_{1}, 0.1 t_{2}, \cdots 0.1 t_{5}, 5^{0}\right)$

Uncertainties of design variables

$$
\begin{aligned}
\delta \vec{M} & =\left(\delta E_{1}^{1}, \delta E_{2}^{1}, \delta G_{12}^{1}, \delta E_{1}^{2}, \delta E_{2}^{2}, \delta G_{12}^{2}\right) \\
& =(18100,1030,717,7400,7400,455)
\end{aligned}
$$

Material property uncertainty of T300/N5208

and T300/Fiberite 934,

where $W$ : weight $(\mathrm{Kg})$, Eigen: eigenvalue of buckling, S.F: safety factor (fiber strain failure criterion), $S_{e i}^{d}$ : eigenvalue sensitivity with respect to design variable $i(i=1 \sim 6), S_{s i}^{d}$ : sensitivity of safety factor with respect to design variable $i$ $(i=1 \sim 6), S_{e i}^{m}$ : sensitivity of eigenvalue with respect to material property $j(j=1 \sim 6), S_{s i}^{m}$ : sensitivity of safety factor with respect to material property $j(j=1 \sim 6)$.

The MSC/NASTRAN [20], a general purpose finite element code, is used for analysis and optimization. Because the proposed modified constraints cannot fit the standard form of constraint in MSC/NASTRAN, extra efforts are needed for the calculation of the modified constraints [17]. First of all, dummy FEM (Finite Element) models are constructed which differ from the real FEM models only in the design variables or material properties by small amounts. Secondly, the structural responses such as stress, displacement, natural frequency, and strain are calculated for each dummy FEM model and real FEM model. Next, $S_{j i}^{d}$ (the sensitivity of $j$ th inequality constraint $g_{j}$ with respect to $i$ th design variable $x_{i}$ ) and $S_{j i}^{m}$ (the sensitivity of $j$ th inequality constraint $g_{j}$ with respect to $i$ th material property $M_{i}$ ) are found by the following equations:

$$
\begin{gathered}
S_{j i}^{d}=\frac{R_{j d}^{d}-R_{j r}^{d}}{d x_{i}} \\
S_{j i}^{m}=\frac{R_{j d}^{m}-R_{j r}^{m}}{d M_{i}},
\end{gathered}
$$

where $S_{j i}^{d}$ : sensitivity of constraint $g_{j}$ with respect to design variable $x_{i}, S_{j i}^{m}$ : sensitivity of constraint $g_{j}$ with respect to material property $M_{i}, d x_{i}$ : design variable variation between dummy FEM model and real FEM model, $d M_{i}$ : material property variation between dummy FEM model and real FEM model, $R_{j d}^{d}$ : response $R_{j}$ (objective or constraints) of dummy FEM model with fixed material property, $R_{j r}^{d}$ : response $R_{j}$ of real FEM model with fixed material properties, $R_{j d}^{m}$ : response $R_{j}$ of dummy FEM model with fixed design variables, $R_{j r}^{m}$ : response $R_{j}$ of real FEM model with fixed design variables.

Forth and the last, the modified constraints are incorporated into MSC/NASTRAN by "DEQATN", an efficient built-in function of MSC/NASTRAN for equation execution. After incorporating the modified constraint, the normal procedures of optimization of MSC/NASTRAN are used for analysis. Vanderplaats' modified method of feasible direction and optimization with respect to approximate models [20] is used in MSC/NASTRAN. Local optimum is solved for a prescribed initial design in MSC/NASTRAN. The numerical global optimization solution is investigated using MSC/NASTRAN by starting the design task from different points in the design space. 


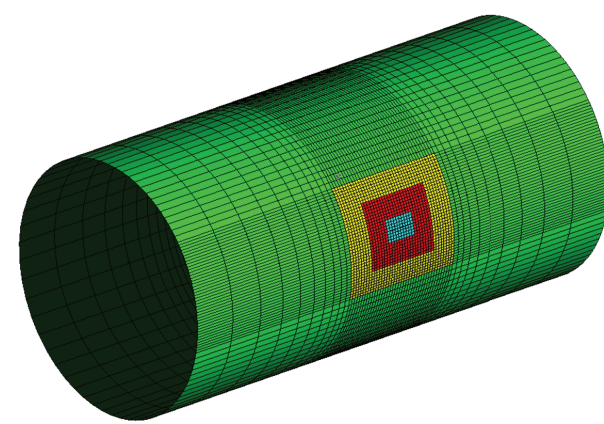

FIGURE 2: FEM model (6008 eight-node composite shell elements).

TABLE 2: Results of mesh size convergence tests.

\begin{tabular}{lcc}
\hline Case & Maximum displacement & Axial stress at inner surface \\
\hline 1 & $5.7101 \mathrm{~mm}$ & $583.49 \mathrm{~N} / \mathrm{mm}^{2}$ \\
2 & $5.7102 \mathrm{~mm}$ & $586.92 \mathrm{~N} / \mathrm{mm}^{2}$ \\
3 & $3.2582 \mathrm{~mm}$ & $405.52 \mathrm{~N} / \mathrm{mm}^{2}$ \\
4 & $3.2551 \mathrm{~mm}$ & $409.84 \mathrm{~N} / \mathrm{mm}^{2}$ \\
\hline
\end{tabular}

Case 1: coarse mesh (total element number: 6008) subjected to uniform pressure loading.

Case 2: finer mesh (total element: 24032) subjected to uniform pressure loading.

Case 3: coarse mesh (total element number: 6008) subjected to local loading. Case 4: finer mesh (total element: 24032) subjected to local loading.

\section{Model Verification}

The FEM model is shown in Figure 2; for an accurate simulation of stress concentration, finer mesh is utilized in the neighborhood of the reinforced patches. 6008 eightnode laminate shell elements are used in the FEM model. In addition to the uniform inner pressure of $4.9 \mathrm{~N} / \mathrm{mm}^{2}$, $1225 \mathrm{~N} / \mathrm{mm}(\mathrm{pr} / 2)$ axial tensile loading is applied at both ends of cylinder to simulate the translated loading from aft and forward dome. The translational and rotational degrees of freedom of a node at left end of cylinder are fixed to prevent rigid body motion for the case of uniform pressure loading, and translational degrees of freedom of nodes at both ends of cylinder are fixed for local loading case. Mesh convergence tests for the initial design condition, that is, $t s=0.5 \mathrm{~mm}, t_{1}=t_{2}=1.5 \mathrm{~mm}, t_{3}=t_{4}=t_{5}=4 \mathrm{~mm}$, and $\theta=30^{\circ}$, have been performed to verify the accuracy of the FEM analysis. The FEM meshes of Figure 2 (coarse mesh) and a finer mesh are used for mesh convergence tests. The element size of the finer mesh is one-half of the corresponding coarse mesh (the total number of element used for the finer mesh is 24032). Table 2 shows the results of mesh convergence tests. The differences between coarse and finer mesh in displacement and stress are small enough to assure the accuracy of the FEM analysis.

A degenerated simple case without metal liner subjected to uniform pressure loading $p$ was used to verify the accuracy of the numerical simulation. Netting analysis [21] that neglects the minor contribution of matrix is a frequently used approximate analytical method for filament wound composite cylinder. The analytical optimal fiber stress in both helical layer and hoop layer is $1.5 \mathrm{pr} / \mathrm{t}$ ( $r$ and $t$ are radius and thickness of cylinder) based on netting analysis [21,22]. Accordingly, the optimal thickness of the composite cylinder with safety factor (SF) of 1.5 is $3.675 \mathrm{~mm}(1.5 * \mathrm{pr} *(\mathrm{SF}) / X=$ $1.5 * 1.5 * 4.9 * 500 / 1500=3.675$, where $X$ is strength in fiber direction of adopted material). The numerically solved optimal thickness for this special case where the contribution of matrix has been dealt is $3.84 \mathrm{~mm}$. The difference between netting analysis and numeral solution is only $4.4 \%$, and the analyzed optimal thicknesses of the three reinforced patches are less than $1 \mathrm{E}-4 \mathrm{~mm}$ for all cases with and without uncertainty, that is, very close to analytical solution of $0.0 \mathrm{~mm}$.

\section{Analysis Results}

The yielding and ultimate strains of SAE4130 are 0.5\% $(980 / 196000)$ and $5 \%$, and the ultimate strains in fiber direction of T300/N5208 and T300/Fiberite 934 are $0.829 \%$ (1500/181000) and $0.674 \%$ (499/74000), respectively. Depending on the utilized design rules, that is, elastic design or plastic design, the allowable strain of SAE4130 may be less or larger than the ultimate strains of T300/N5208 and T300/Fiberite 934. Optimal design for both design rules is carried out to investigate the effect of allowable strain of SAE4130 on optimal designs.

Equation (11) for continuous side constraints of thickness of each sublaminate is based on the assumption of continuous design variables. Practically, the thickness of each sublaminate is discrete, it can only be $N(N=1,2, \ldots)$ times of nominal ply thickness (the discrete sublaminate thicknesses are $0.2 \mathrm{~mm}, 0.4 \mathrm{~mm}, \ldots$ ). Rounding processes [20] are often used to obtain the most suitable discrete design variables after the optimal design has been obtained from the continuous design variable condition. There are four rounding processes in MSC/NASTRAN, namely, rounding up to the nearest discrete design variables, rounding off to the nearest discrete design variable, conservative discrete design (CDD), and design of experiments (DOE). The first two methods require no new analysis but they are too rough for an accurate design optimization of discrete design variables. DOE makes use of concept of orthogonal arrays that provide much more efficient rounding process than CDD for the cases of large design variables [20]; hence, DOE is used in the analysis.

Table 3 (for continuous design variable) and Table 4 (for discrete design variable) show the optimal design variables of 4 uncertainty conditions, that is, no uncertainty (case 1), only property uncertainties (case 2), only material uncertainties (case 3), and both property and material uncertainties (case 4). If plastic design of metallic liner is utilized, the allowable strain of metallic liner is much larger than composite material. The optimal weights for $t s$ equals to $0.05 \mathrm{~mm}, 0.5 \mathrm{~mm}, 1.0 \mathrm{~mm}$, and $1.5 \mathrm{~mm}$ are shown in Table 5 (for continuous design variable) and Table 6 (for discrete design variable) for case of plastic design. It is found that the optimal designs are significantly affected by both property and material uncertainties, and optimal weights increase 
TABLE 3: Optimal continuous design variables for case of $0.5 \mathrm{~mm}$ SAE4130 liner when plastic design of SAE4130 liner is utilized.

\begin{tabular}{lcccc}
\hline Design variable & No uncertainty & Property uncertainties & Material uncertainties & Property and material uncertainties \\
\hline$t_{1}(\mathrm{~mm})$ & 1.33 & 0.81 & 1.76 & 1.52 \\
$t_{2}(\mathrm{~mm})$ & 0.60 & 3.02 & 0.74 & 3.16 \\
$t_{3}(\mathrm{~mm})$ & 6.00 & 5.99 & 6.00 & 6.00 \\
$t_{4}(\mathrm{~mm})$ & 4.82 & 5.99 & 5.72 & 6.00 \\
$t_{5}(\mathrm{~mm})$ & 6.00 & 6.00 & 6.00 & 6.00 \\
$\theta(\mathrm{deg})$ & 55.5 & 42.5 & 53.6 & 51.7 \\
\hline
\end{tabular}

TABLE 4: Optimal discrete design variables for case of $0.5 \mathrm{~mm}$ SAE4130 liner when plastic design of SAE4130 liner is utilized.

\begin{tabular}{lcccc}
\hline Design variable & No uncertainty & Property uncertainties & Material uncertainties & Property and material uncertainties \\
\hline$t_{1}(\mathrm{~mm})$ & 1.40 & 1.00 & 1.80 & 1.60 \\
$t_{2}(\mathrm{~mm})$ & 0.60 & 3.00 & 0.80 & 3.20 \\
$t_{3}(\mathrm{~mm})$ & 6.00 & 6.00 & 6.00 & 6.00 \\
$t_{4}(\mathrm{~mm})$ & 5.00 & 5.80 & 5.60 & 6.00 \\
$t_{5}(\mathrm{~mm})$ & 6.00 & 6.00 & 6.00 & 6.00 \\
$\theta(\mathrm{deg})$ & 55.5 & 42.5 & 53.6 & 51.7 \\
\hline
\end{tabular}

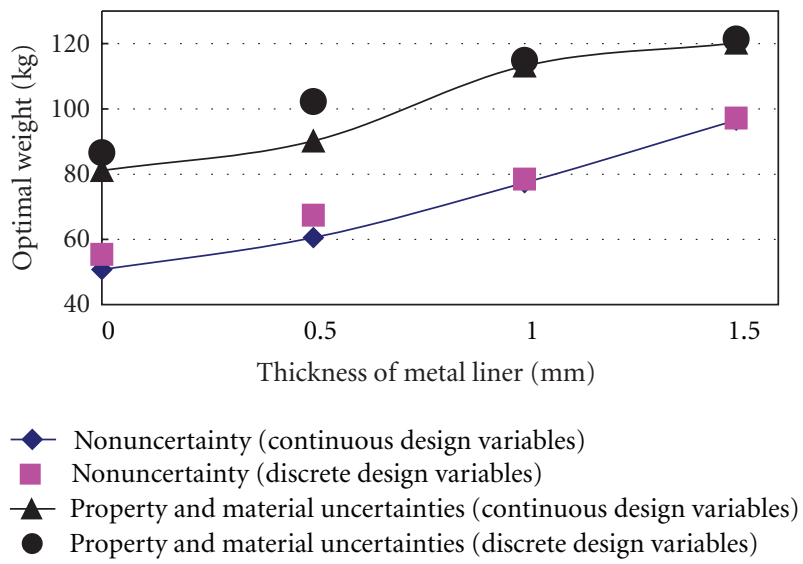

Figure 3: Optimal weight versus thickness of SAE4130 liner (plastic design of SAE4130 liner is utilized).

with uncertainties. The decreasing effect of uncertainties on optimal weight with increasing ts can also be found. Smaller $t s$ means larger ratio of composite material to metal, and the uncertainties of T300/N5208 and T300/Fiberite 934 play more significant role for the whole structure in these cases. Comparing with their nonuncertainty counterparts, the optimal weight increases up to $59.94 \%$ and $56.19 \%$, respectively, for continuous design variable and discrete design variable cases when ts approximates zero. These values decrease to $24.45 \%$ and $24.95 \%$ for cases of $t$ s equals to $1.5 \mathrm{~mm}$. The optimal weight versus ts curve is shown in Figure 3, and the optimal weight versus uncertainty curves for the above-mentioned 4 uncertainty conditions are shown in Figures 4, 5, and 6. The decrease of the optimal weight with ts implies that sufficiently small ts should be utilized if it is compatible with the manufacturing process and the non-leakage constraint. For plastic design case, the allowable strain of metallic liner is much larger than

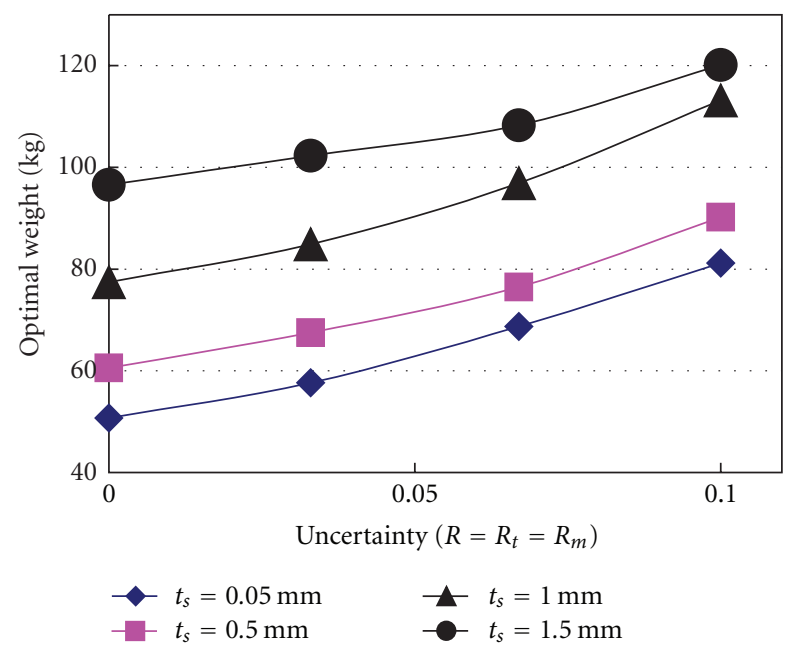

FIGURE 4: Optimal weight versus material and ply thickness uncertainties (plastic design of SAE4130 liner is utilized, continuous design variables, ply orientation uncertainty equals to $50 R_{t}$ ).

composite material, the composite material is always more critical than the metallic linear; thicker SAE4130 means more conservative design for the metallic liner; hence, the optimal weight increases with the thickness of metallic liner.

For cases of elastic design of SAE4130 where the stress of SAE4130 must be kept below its yielding stress, the allowable strain of SAE4130 is less than the allowable strains of T300/N5200 and T300/Fiberrite 934. The optimal weight versus $t$ s curve and the optimal weight versus uncertainty curve are shown in Figure 7 and Figure 8, respectively, for elastic design cases. For larger $t s$ ( $t s \geqq 0.5 \mathrm{~mm}$ ), the safety factor of metallic liner is larger than that of the composite material; hence, the optimal weight increases with $t s$. For cases with very thin metallic liner, the metallic liner is more critical than the composite material. To assure the safety of 
TABLE 5: Optimal weights for continuous design variable case when plastic design of SAE4130 liner is utilized.

\begin{tabular}{lccc}
\hline$t s(\mathrm{~mm})$ & Uncertainty condition & Optimal weight $(\mathrm{Kg})$ & Optimal weight with uncertainty/optimal weight without uncertainty $(\%)$ \\
\hline 0.05 & None & 50.75 & $100 \%$ \\
0.05 & $R_{t}=0.1, R_{m}=0.0$ & 63.97 & $126.05 \%$ \\
0.05 & $R_{t}=0.0, R_{m}=0.1$ & 64.11 & $126.32 \%$ \\
0.05 & $R_{t}=0.1, R_{m}=0.1$ & 81.17 & $159.94 \%$ \\
0.5 & None & 60.58 & $100 \%$ \\
0.5 & $R_{t}=0.1, R_{m}=0.0$ & 74.54 & $123.04 \%$ \\
0.5 & $R_{t}=0.0, R_{m}=0.1$ & 70.73 & $116.75 \%$ \\
0.5 & $R_{t}=0.1, R_{m}=0.1$ & 90.22 & $148.92 \%$ \\
1.0 & & 77.43 & $100 \%$ \\
1.0 & $R_{t}=0.1, R_{m}=0.0$ & 89.26 & $115.27 \%$ \\
1.0 & $R_{t}=0.0, R_{m}=0.1$ & 87.93 & $113.56 \%$ \\
1.0 & $R_{t}=0.1, R_{m}=0.1$ & 113.14 & $146.11 \%$ \\
1.5 & & None & $100 \%$ \\
1.5 & $R_{t}=0.1, R_{m}=0.0$ & 106.60 & $110.40 \%$ \\
1.5 & $R_{t}=0.0, R_{m}=0.1$ & 105.31 & $109.06 \%$ \\
1.5 & $R_{t}=0.1, R_{m}=0.1$ & 120.17 & $124.45 \%$ \\
\hline
\end{tabular}

$R_{t}$ : ratio of ply thickness uncertainty to ply thickness.

$R_{m}$ : ratio of material property uncertainty to nominal material property.

Ply orientation uncertainty equals to $50 R_{t}$.

TABLE 6: Optimal weights for discrete design variable case when plastic design of SAE4130 liner is utilized.

\begin{tabular}{lccc}
\hline$t s(\mathrm{~mm})$ & Uncertainty condition & Optimal weight $(\mathrm{Kg})$ & Optimal weight with uncertainty/optimal weight without uncertainty $(\%)$ \\
\hline 0.05 & None & 55.45 & $100 \%$ \\
0.05 & $R_{t}=0.1, R_{m}=0.0$ & 72.69 & $131.09 \%$ \\
0.05 & $R_{t}=0.0, R_{m}=0.1$ & 68.22 & $123.03 \%$ \\
0.05 & $R_{t}=0.1, R_{m}=0.1$ & 86.61 & $156.19 \%$ \\
0.5 & None & 61.97 & $100 \%$ \\
0.5 & $R_{t}=0.1, R_{m}=0.0$ & 78.17 & $126.14 \%$ \\
0.5 & $R_{t}=0.0, R_{m}=0.1$ & 72.12 & $116.37 \%$ \\
0.5 & $R_{t}=0.1, R_{m}=0.1$ & 92.24 & $148.84 \%$ \\
1.0 & & 78.52 & $100 \%$ \\
1.0 & $R_{t}=0.1, R_{m}=0.0$ & 90.78 & $115.61 \%$ \\
1.0 & $R_{t}=0.0, R_{m}=0.1$ & 88.71 & $112.97 \%$ \\
1.0 & $R_{t}=0.1, R_{m}=0.1$ & 114.86 & $146.28 \%$ \\
1.5 & & None & $100 \%$ \\
1.5 & $R_{t}=0.1, R_{m}=0.0$ & 97.18 & $110.48 \%$ \\
1.5 & $R_{t}=0.0, R_{m}=0.1$ & 107.37 & $110.37 \%$ \\
1.5 & $R_{t}=0.1, R_{m}=0.1$ & 107.26 & $124.95 \%$ \\
\hline
\end{tabular}

$R_{t}$ : ratio of ply thickness uncertainty to ply thickness.

$R_{m}$ : ratio of material property uncertainty to nominal material property.

Ply orientation uncertainty equals to $50 R_{t}$.

metallic liner for case with thin metallic liner, it is necessary to enlarge the thickness of composite to reduce the stress of metallic liner; hence, the optimal weight decreases with ts if $t s<0.5 \mathrm{~mm}$. The minimal optical weight occurs at $t s=0.5 \mathrm{~mm}$ for case of elastic design.

\section{Concluding Remarks}

By adding extra terms associated with sensitivities and uncertainties to traditional constraints, the robust optimal design of composite cylinder with metallic liner subjected to uniform pressure and local loading is investigated. Degenerated simple case is used to verify the effectiveness and accuracy of the developed analysis method. Ply thickness and orientation uncertainties and material property uncertainties are found to have strong effect on the optimal design of composite cylinder and that the optimal weight increases significantly with uncertainties is shown as expected. Comparing with their non-uncertainty counterparts, the most significant effect of uncertainty among the analyzed examples shows 


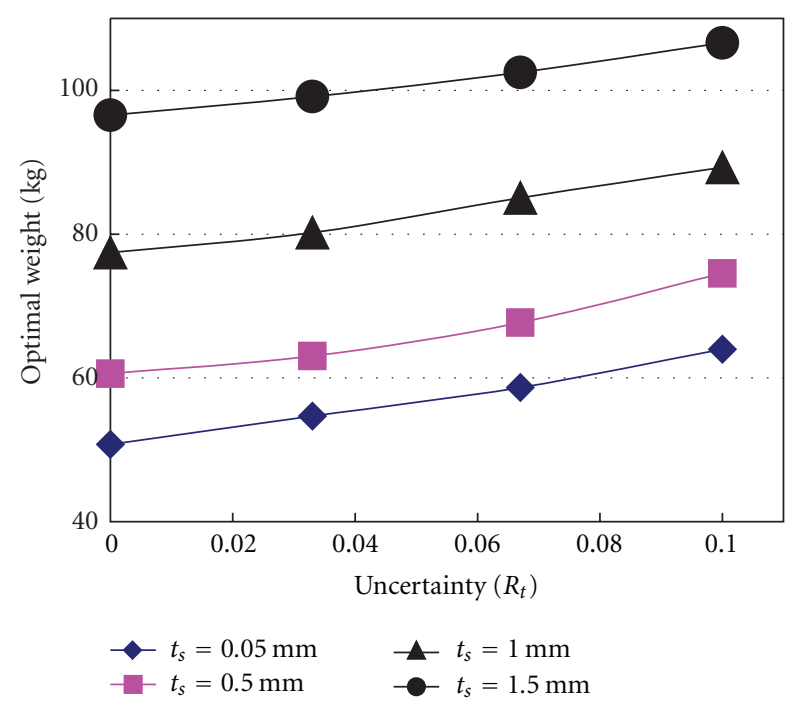

FIGURE 5: Optimal weight versus ply thickness uncertainty (plastic design of SAE4130 liner is utilized, continuous design variables, ply orientation uncertainty equals to $50 R_{t}$ ).

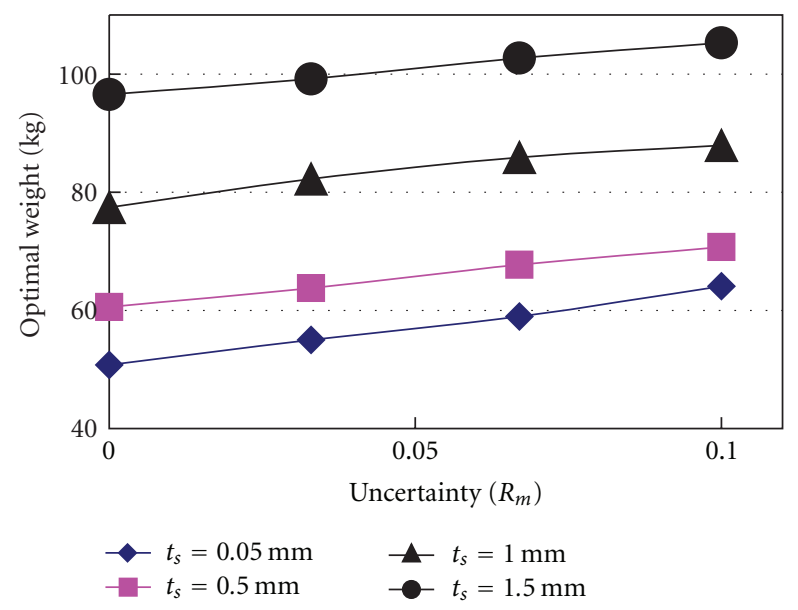

Figure 6: Optimal weight versus material uncertainty (plastic design of SAE4130 liner is utilized, continuous design variables).

the optimal weights increase up to $59.94 \%$ and $56.19 \%$, respectively, for continuous design variable and discrete design variable cases. The significant effect of ply thickness, orientation, and material property uncertainties on optimal weight is one of main reasons why higher safety factor is necessary for composite cylinder if effect of uncertainty is neglected. It is also found that the optimal thickness of metallic liner is affected by the utilized design rule of metallic liner. For plastic design, the thickness of metallic liner should be kept as small as possible for a minimal weight optimal design provided that manufacturing and non-leakage constraints can be met. On the contrary the optimal thickness of metallic liner depends on the relative ratio of allowable strain of metallic liner and composite material if elastic design is used.

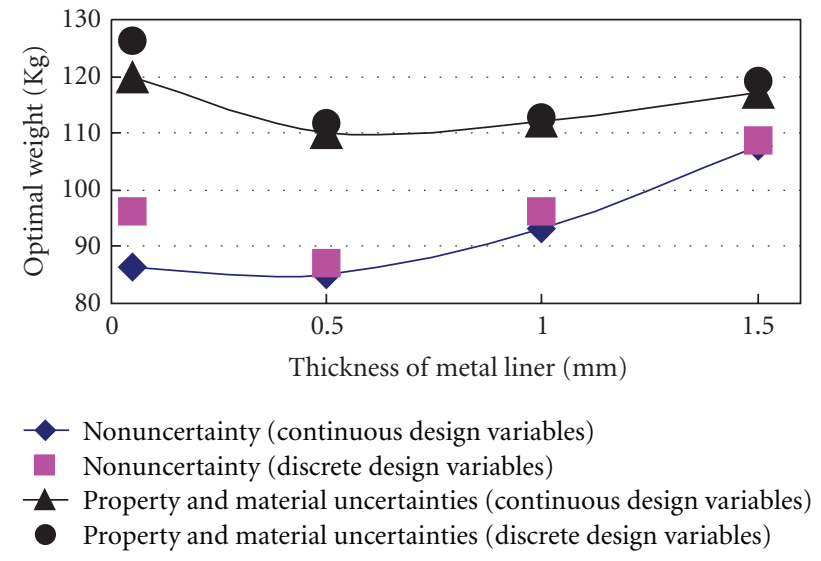

FIGURE 7: Optimal weight versus ply thickness of metal liner (elastic design of SAE4130 liner is utilized).

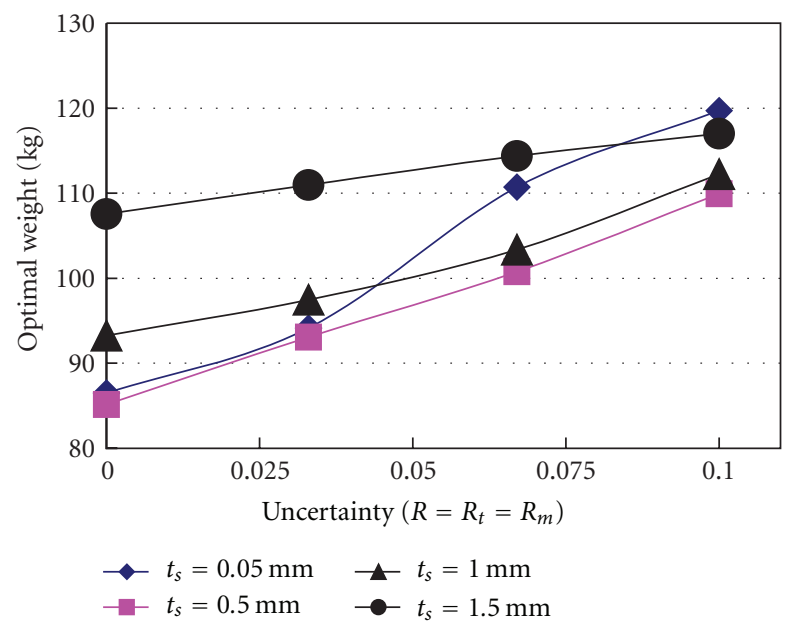

Figure 8: Optimal weight versus material and ply thickness uncertainties (elastic design of SAE4130 liner is utilized, continuous design variables, ply orientation uncertainty equals to $50 R_{t}$ ).

\section{References}

[1] S. W. Tsai, Composite Design, Thick Composite, Paris, France, 4th edition, 1988.

[2] C. Becker-Willinger, P. Kalmes, P. Herbeck-Engel, and M. Veith, "Micro-patternable hybrid nanocomposites with tailorable mechanical and thermomechanical properties," in Advanced Fabrication Technologies for Micro/Nano Optics and Photonics III, vol. 7591 of Proceedings of SPIE, San Francisco, Calif, USA, January 2010.

[3] P. B. Gning etc, "Prediction of damage in composite cylinders after impact," Journal of Composite Materials, vol. 39, no. 10, pp. 917-928, 2005.

[4] H. E. Soliman and R. K. Kapania, "Probability of fatigue of composite cylinders subjected to axisymmetrical loading," AIAA Journal, vol. 43, no. 6, pp. 1342-1348, 2005.

[5] J. S. Park, C. S. Hong, C. G. Kim, and C. U. Kim, "Analysis of filament wound composite structures considering the change of winding angle through thickness direction," Composite Structure, vol. 55, no. 1, pp. 63-71, 2002. 
[6] O. Dolit, E. Carrera, and T. Wallmorsporger, "Design modeling and experiments of adaptive structure and smart systems III," Mechanics of Advanced Materials and Structures, vol. 18, no. 7, pp. 467-468, 2011.

[7] C. W. Kong, J. H. Yoon, Y. S. Jang, and Y. M. Yi, "Design of composite pressure vessel with metallic and plastic liners," Key Engineering Materials, vol. 261-263, pp. 1505-1510, 2004.

[8] J. Q. Zhao, R. G. Wang, X. D. He, and W. B. Liu, "Strain monitoring of composite pressure vessel with thin metal liner using fiber Bragg grating," in 2nd International Conference on Smart Materials and Nanotechnology in Engineering, vol. 7493 of Proceedings of SPIE, Weihai, China, July 2009.

[9] P. J. Nunes, F. J. Silva, and J. P. Antunes, "Demestic gas vessel manufactured by using hybrid steel glass reinforced thermoplastic matrix solution," in Proceedings of the ASME Pressure Vessel and Piping Conference, vol. 6, parts A \& B, 2010.

[10] X. Y. Qu, R. T. Haftka, and T. F. Johnson, "Deterministic and reliability-based optimization of composites for cryogenic environments," AIAA Journal, vol. 41, no. 10, pp. 2029-2036, 2003.

[11] M. Y. Cho and S. Y. Rhee, "Optimization of laminate with free edges under bounded uncertainty subject to extension, bending and twisting," Internal Journal of Solids and Structures, vol. 41, no. 1, pp. 227-245, 2004.

[12] C. Zang, M. I. Friswell, and J. E. Mottershead, "A review of robust optimal-design and its application in dynamics," Computers and Structures, vol. 83, no. 4-5, pp. 315-326, 2005.

[13] M. Lombardi and R. T. Haftka, "Anti-optimization technique for structural design under load uncertainties," Computer Methods in Applied Mechanics and Engineering, vol. 157, no. 1-2, pp. 19-31, 1998.

[14] A. R. Defaria, "Buckling optimization and anti-optimization of composite plates: uncertain loading combination," International Journal For Numerical Methods in Engineering, vol. 53, no. 3, pp. 719-732, 2002.

[15] I. Elishakoff, Y. W. Li, and J. H. Starnes Jr., "A deterministic method to predict the effect of unknown-but-bounded elastic moduli on the buckling of composite structures," Computer Methods in Applied Mechanics and Engineering, vol. 111, no. 1-2, pp. 155-167, 1994.

[16] I. Elishakoff, "Essay on uncertainties in elastic and viscoelastic structures: from A. M. Freudental's criticisms to modern convex modeling," Computers and Structures, vol. 56, no. 6, pp. 871-895, 1995.

[17] Y. S. Liao and C. Y. Chiou, "Robust optimum designs of fiber reinforced composites using constraints with sensitivity," Journal of Composite Materials, Journal of Composite Materials, vol. 40, no. 22, pp. 2067-2081, 2006.

[18] J. D. Mathias, X. Balandraud, and M. Grediac, "Appling a genetic algorithm to the optimization of composite patches," Computers and Structures, vol. 84, no. 12, pp. 823-834, 2006.

[19] Department of Defense, Military Handbook 5 G: Metallic Materials and Elements for Aerospace Vehicle Structures, Department of Defense, Washington, DC, USA, 1994.

[20] G. J. Moore, MSC.NASTRAN Design Sensitivity and Optimization User's Guide, 2004.

[21] J. T. Evans and A. G. Gibson, "Composite angle-ply laminates and netting analysis," Proceedings of Royal Society of London Series A, vol. 458, no. 2028, pp. 3079-3088, 2002.

[22] A. Puck and H. Schurmann, "Failure analysis of FRP laminates by means of physically based phenomenological models," Composites Science and Technology, vol. 62, no. 12-13, pp. 1633-1662, 2002. 

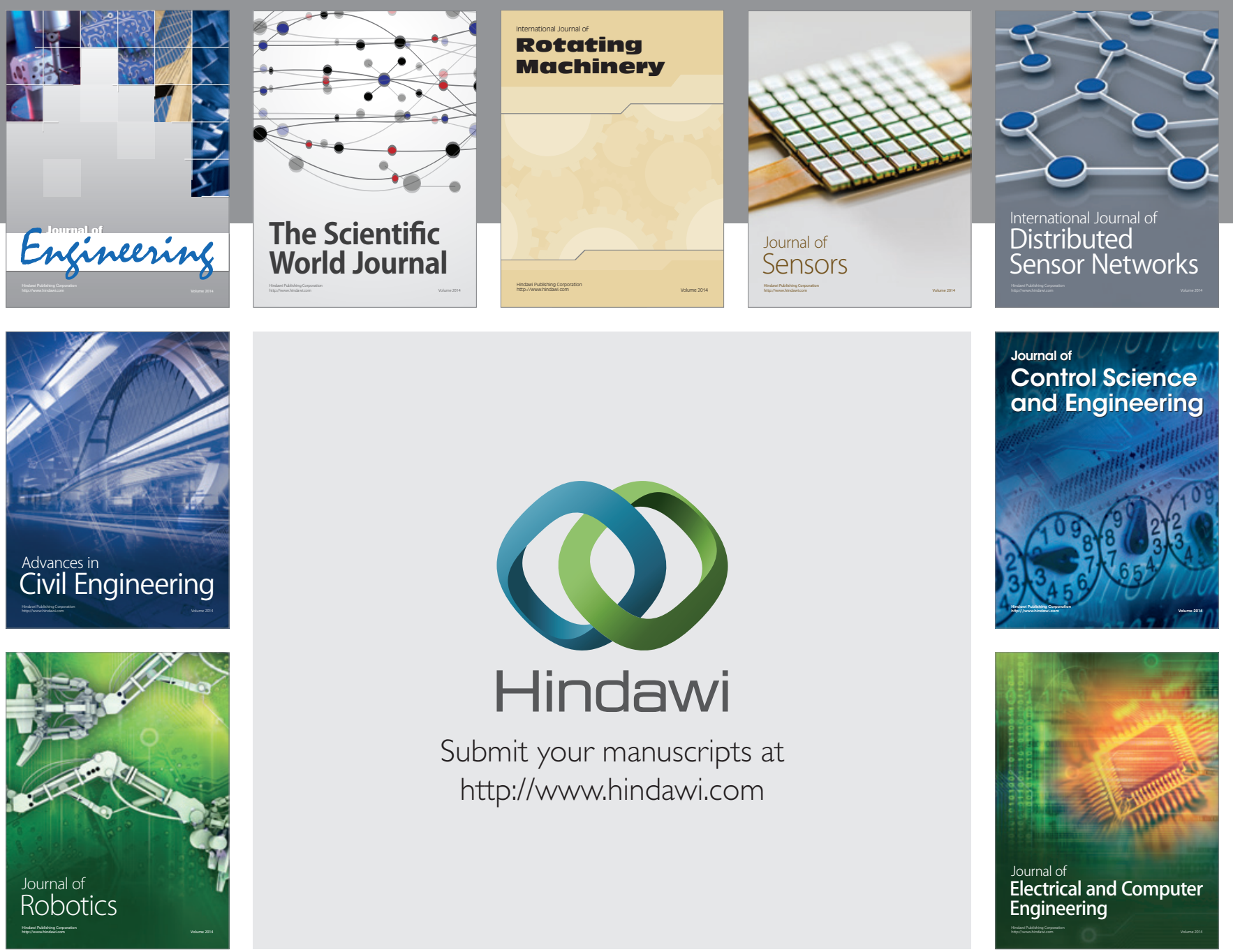

Submit your manuscripts at

http://www.hindawi.com
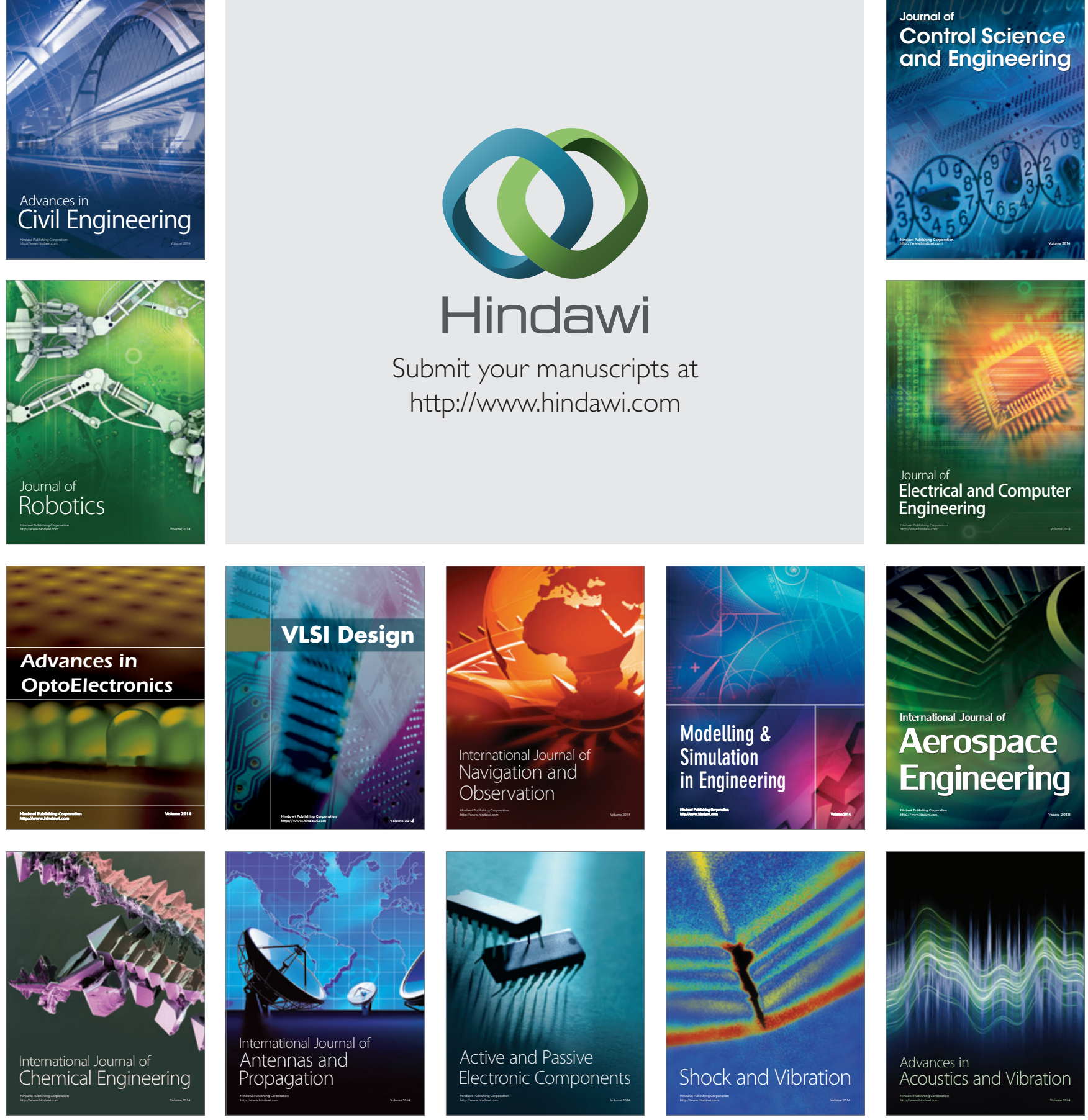